\title{
Notas para una historia de las instituciones argentinas de formación de docentes en Educación Física durante el siglo xx
}

Alejo Levoratti y Pablo Scharagrodsky

\section{RESUMEN}

Este ensayo busca analizar los procesos de institucionalización de la formación de los profesores de Educación Física en Argentina durante el siglo XX. Para ello relevamos las instancias de creación de las instituciones educativas y las principales líneas rectoras de la formación disciplinar. Con base en ello identificamos cuatro momentos de la matriz instruccional, en donde se procuró instituir determinadas concepciones disciplinares, modelos formativos y campos de acción profesional. Ello encontró sustento en diversas disciplinas científicas, la participación de actores sociales inscritos en múltiples tramas y los lineamientos de las políticas educativas destinadas al nivel superior.

Palabras clave: educación física, formación docente, instituciones, Argentina.

\section{Alejo Levoratti}

levoratti@gmail.com

Argentino. Doctor en Ciencias Sociales y Humanas por la Universidad Nacional de Quilmes (UNQ), Argentina. Investigador del Consejo Nacional de Investigaciones Científicas y Técnicas (CONICET), Argentina; Docente de la Universidad Nacional de La Plata (UNLP), Argentina. Temas de investigación: estudios sociohistóricos sobre la educación física y el deporte; procesos de formación y configuración profesional de los profesores de educación física; estudios antropológicos sobre el deporte y la educación física. ORCID: http://orcid.org/0000-0003-2749-0321. 
Notas para uma história das instituições argentinas de formação de docentes em Educação Física durante o século $\mathrm{xx}$

\section{RESUMO}

Este ensaio procura analisar os processos de institucionalização da formação dos professores de Educação Física na Argentina durante o século XX. Para isso relevamos as instâncias de criação das instituições educativas e das principais linhas reitoras da formação disciplinar. Com base nisso identificamos quatro momentos da matriz instrucional, onde se procurou instituir determinadas concepções disciplinares, modelos formativos e campos de ação profissional. O anterior encontrou sustento em diversas disciplinas científicas, na participação de atores sociais inscritos em múltiplos tramas e nos alinhamentos das políticas educativas destinadas ao nível superior.

Palavras chave: educação física, formação docente, instituições, Argentina.

\section{Notes for a history of Argentine teacher training institutions in Physical Education during the 20th century}

\section{ABSTRACT}

This article offers an analysis of the processes of institutionalization of Physical Education teacher training in Argentina during the twentieth century. In order to do so, the authors investigated the instances of creation of educational institutions and the main guiding lines of disciplinary training. Based on this, they identified four moments of the instructional matrix, during which certain disciplinary conceptions, training models and professional action fields were established and found support in various scientific disciplines, the participation of social actors inscribed in multiple plots and the guidelines of educational policies for higher education.

Key words: physical education, teacher training, institutions, Argentina. 


\section{Introducción}

En Argentina, durante las dos últimas décadas del siglo XIX, quienes ejercieron el rol de docentes en la disciplina educativa asociada con lo que hoy conocemos como Educación Física (gimnástica, gimnasia, ejercicios físicos, ejercicios militares, higiene física, etcétera), fueron grupos de idóneos, sportsman o expertos en alguna actividad física (algún deporte, cierto tipo de gimnasia, arte circense, masajistas, etcétera), extranjeros con alguna acreditación institucional o amplia reputación, médicos interesados en la cultura física, militares vinculados con algunas prácticas específicas (como tiro, gimnasia militar, batallones escolares), pedagogos o, fundamentalmente, maestros y/o profesores egresados de las escuelas normales creadas en el país en 1870.

Sin embargo, algo sustancial cambió entre finales del siglo XIX y principios del XX. Por primera vez, el Estado educador argentino - en plena constitución-(Lionetti, 2007; Alliaud, 2007; Pineau, 2014) centró su atención en la formación de "educadores físicos" para el ámbito escolar y civil diferenciándolo del maestro "generalista" (del maestro y profesor normalista).

Este proceso se produjo en un contexto internacional en donde las instituciones de formación docente en la especialidad de Educación Física en varios países europeos (Suecia, Francia, Alemania, España, Suiza y otros) o en los Estados Unidos (EUA) (Vigarello, 2005; Ljunggren, 2011; Mayoral, 2012; Torres, 2011) ya estaban funcionando y fabricando el nuevo oficio, y consolidando formalmente un tipo de intervención especifica vinculada no sólo con la educación de los cuerpos en las instituciones educativas, sino también en instituciones y espacios de la sociedad civil (clubes deportivos, clubes atléticos, clubes de barrio, clubes gremiales, empresariales e industriales, sociedades de fomento, sociedades militares, de beneficencia, de socorros mutuos, recreos infantiles, etcétera), en el marco del nuevo proyecto higiénico-recreacional moderno, del aumento y diversificación del uso del tiempo de ocio y de la amplia difusión del modelo de vida activo, saludable $\mathrm{y}$ al aire libre iniciado a mediados del siglo XIX y potenciado en varios países de occidente en las primeras décadas del siglo XX.

Argentina, como muchos países de América latina (Chile, Brasil, Uruguay, Colombia, México, entre otros) (González de Álvarez, 2012) se sumaron a este proceso de carácter trasnacional vinculado con la constitución de instituciones particulares de educación, siendo Chile y Argentina los primeros países latinoamericanos en materializar dispositivos de formación en Educación Física en los primeros años del siglo XX (Cornejo et al., 2011; Aisenstein, 2007).

Estos espacios educativos, lentamente, inventaron el oficio de "educador físico" a partir de una serie de características específicas que fueron variando a lo largo del tiempo: acreditación formal, transmisión de determinados saberes, habilidades y competencias, definición de un tipo particular de intervención, construcción de un tipo de identidad profesional, reconocimiento social de su expertiz, circulación de ciertos rituales, ejercicio legítimo y, en lo posible, monopólico de la actividad, organización de asociaciones vinculadas con la cultura física, etcétera.

Teniendo en cuenta todo ello, a partir de los aportes generales de la historia social y cultural y las contribuciones particulares de la historia social de la educación, este trabajo tiene como objetivo central identificar y mapear en forma "panorámica" los diversos centros de formación que se constituyeron en Argentina durante el largo siglo XX (sus características, lógicas, enfrentamientos y, muy especialmente, sus diferenciales capacidades para ejercer poder, influencia y autoridad), estableciendo ciertos momentos de ruptura y discontinuidad en esta historia de larga duración. Nuestra hipótesis es que durante buena parte del siglo XX y hasta por lo menos la década de los sesenta y con fuertes rupturas en los ochenta y noventa, el Instituto Nacional de Educación Física (INEF) creado en la primera década en la 
capital argentina, fue el modelo a seguir por la mayoría de las instituciones constituidas posteriormente. Más allá de las traducciones, resignificaciones y resistencias de parte de los actores locales en las nuevas instituciones de formación que le sucedieron al INEF, de los conflictos al interior de cada institución y de la co-presencia conflictiva de otros formatos de educación relacionados con la especialidad, el INEF, como formato instituido, se convirtió en lo instituyente y, en muchos sentidos, en un modelo rector a seguir para el resto de las instituciones de formación en Educación Física de la República Argentina. De alguna manera, el INEF emergió y se constituyó en un "modelo" a imitar por la mayoría de las instituciones de formación creadas posteriormente. De esta manera, se definió una matriz instruccional ejemplar y ejemplificadora, así como la única posible y deseable con relación al tipo de formación dictando planes y programas de estudio específicos, seleccionado ciertos contenidos, prescribiendo determinada bibliografía, estableciendo formas de ingreso, exámenes particulares, rituales típicos y distintivos, tipos de titulaciones, formas de inserción laboral, una particular cultura institucional y modos distintivos de articulación con organismos estatales relacionados con la Educación Física. Vale decir, el INEF se convirtió en la institución que monopolizó, no sin tensiones y disputas, la producción, transmisión, distribución y organización de la cultura corporal escolar adecuada y deseable, y el tipo "ideal" del buen profesor en Educación Física en Argentina en buena parte del siglo XX. Para su estudio trabajamos con fuentes primarias y secundarias. En este sentido se recopilaron documentos oficiales entre los que se encontraron decretos, resoluciones, planes de estudio y programas de asignaturas. Además, se produjo un relevamiento donde se procuró identificar la modalidad de inscripción dentro del sistema de educación superior y el periodo en que se dictó la carrera.

En la compleja y heterogénea cartografía de instituciones de formación en el arte de enseñar la
Educación Física identificamos, a grandes rasgos, cuatro grandes momentos o etapas con tensiones internas y subperiodos en cada una de ellas, producto de cambios, traducciones locales, resistencias, micromatices o pequeñas discontinuidades.

Un primer periodo fue el momento en que se inventó una determinada tradición sobre el oficio de enseñante en la Educación Física en las primeras tres décadas y media del siglo XX, abarcando el periodo comprendido de 1901 a 1938. Un segundo periodo fue producto de la estabilización y consolidación de la matriz instruccional de formación en Educación Física con un fuerte impacto nacional entre fines de los años treinta hasta principios de los años sesenta. Un tercer periodo, entre los años sesenta, setenta y principios de los ochenta, el cual se generó a partir de la propagación de las matrices de formación en la Educación Física en varias ciudades y provincias argentinas siguiendo, con matices, el modelo del INEF de los periodos anteriores. Este periodo consolidó una serie de rituales, prácticas, lógicas y sentidos, aunque, al mismo tiempo, los mismos fueron re-significados regionalmente por los actores y las tensiones locales y provinciales, y por nuevas perspectivas en la formación docente. Por último, el cuarto periodo aconteció a partir de mediados de los años ochenta y, muy especialmente, la década de los noventa, en el marco de importantes cambios en las políticas educativas, con nuevos marcos epistémicos y regulatorios, generando nuevos sentidos en la matriz instruccional en algunos centros de formación y un aumento exponencial de los mismos en términos generales, pasando de ser aproximadamente 30 a principios de los ochenta a casi 200 en la actualidad, el 88\% de ellos pertenecientes al sistema de educación superior no universitario.

En este periodo la matriz de formación de los INEF comenzó a ser desplazada en términos de poder, reconocimiento, prestigio y autoridad por otras instituciones de formación, especialmente las universitarias, destacándose muy especialmente 
el Profesorado Universitario de Educación Física (PUEF) de la Universidad Nacional de la Plata (UNLP), con su protagonismo como centro productor y difusor de saberes específicos, con una sustantiva producción académica y con una cada vez mayor influencia de sus egresados y docentes en espacios de formación y en la definición de ciertas políticas vinculados al campo. Asimismo, en este periodo se visibilizó el paulatino crecimiento de instituciones privadas de formación, llegando en la actualidad a representar el 56\% de la totalidad de las propuestas educativas. Aunque la primera propuesta privada se abrió en el país recién en 1971, su proliferación se produjo a partir de 1983. Esto construyó un espacio social con una intensa competencia entre las distintas instituciones de formación terciaria y universitaria, tanto públicas como privadas, que se constituyeron en los últimos 20 años.

\section{La etapa fundacional en la formación de "educadores físicos" o acerca de una nueva "tradición inventada"}

El 20 de diciembre de 1901, en presencia del inspector general de Enseñanza Secundaria y Normal Pablo Pizzurno y bajo la dirección de Enrique Romero Brest, ${ }^{1}$ comenzó el primer curso estatal "temporario de Ejercicios Físicos" en la capital Argentina. En estos primeros cursos, bajo el normalismo, el pan-higienismo y el cientificismo, se forjó lentamente el oficio de educador físico y se construyó la tradición "romerista" fabricada e inventada ${ }^{2}$ en el campo de la Educación Física.
Estos primeros cursos esbozaron los temas, materias, contenidos, argumentaciones y bibliografía que serían utilizados en décadas posteriores. En estos cursos se destacó la influencia higienista y fisiologista, muy especialmente francesa, a la hora de definir los saberes y prácticas necesarios para formar a todo/a buen "educador físico". El análisis anatómico de los movimientos y los principios de la fisiología del ejercicio sentaron las bases epistémicas y científicas para la formación de cualquier "buen" profesor/ $\mathrm{a}^{3}$ en educación física.

Los primeros cursos inventaron la tradición "romerista" retomando en forma ecléctica ciertos sistemas decimonónicos de gimnasia, resignificándolos a partir de necesidades y conflictos locales y fabricando una nueva narrativa sobre la educación corporal: el Sistema Argentino de Educación Física (SAEF). El SAEF, vigente en escuelas y colegios hasta 1938, fue producto de una combinación de axiomas, principios y características provenientes de distintos sistemas europeos ya existentes. Fue un sistema ecléctico que cuestionó al sistema francés y alemán - a tono con la época y con la influencia fisiológica francesa- y se nutrió de aspectos medulares de otros sistemas, especialmente del sueco por su 'cientificidad', su capacidad higiénica, respiratoria y descongestionante y, en menor medida, del inglés por el predominio de los ejercicios de sofocación y el desarrollo de la emotividad; coexistiendo cuestiones fisiológicas vinculadas con la gimnasia, pero incorporando a los juegos y - con determinados

\footnotetext{
${ }^{1}$ El doctor Enrique Romero Brest (1873-1958) — acompañado por un heterogéneo grupo de trabajo - se convirtió en una de las figuras centrales de la Educación Física en Argentina en las primeras tres décadas del siglo XX. Dirigió el primer dispositivo civil de formación docente de la especialidad, generó una propuesta de educación corporal para las escuelas y colegios argentinos (Sistema Argentino de Educación Física), creó y administró la primera revista de la especialidad, tuvo directa intervención en la definición de políticas educativas relacionadas con la especialidad y se convirtió en una referencia epistémica ineludible en el campo de la educación y la Educación Física argentina y latinoamericana.

${ }^{2}$ Hobsbawm sostiene que el término tradición inventada "incluye tanto las tradiciones realmente inventadas, construidas y formalmente instituidas, como las surgidas de una manera menos identificable en un periodo breve y datable — en cosa de algunos años, tal vez - y establecidas con gran rapidez [...] se considera que la tradición inventada significa un conjunto de prácticas, por lo general gobernadas por reglas abiertas o tácitamente aceptadas y de naturaleza ritual o simbólica que procuran poner en circulación ciertos valores y normas de comportamiento mediante la repetición, que implica automáticamente la continuidad con el pasado. En realidad, siempre que sea posible intentan establecer la continuidad con un pasado histórico conveniente" (Hobsbawm, 1985: 1).

${ }^{3}$ Más del $80 \%$ de los egresados del periodo fueron mujeres.
} 
recaudos - a ciertos sports provenientes del sistema inglés (Scharagrodsky, 2015). En este sentido, la definición del sistema como "argentino" fue menos un aspecto novedoso y original y más una estrategia retórica para afirmar su propuesta en Argentina y darle visibilidad pública — léase escolar-, ya que sus fundamentos teóricos y su propuesta práctica fueron centralmente tomados del sistema sueco.

Los primeros cursos se repitieron en los años siguientes hasta que en 1906 se creó el curso normal de educación física. Este nuevo status se elevó tres años después alcanzando la categoría de Escuela Normal de Educación Física. Ya en 1912 la Escuela, con mayores recursos materiales y simbólicos, se convirtió en Instituto Nacional Superior de Educación Física (INEF), siendo el único centro de formación civil del país. El mismo se consolidó durante los años veinte y treinta alcanzando al final de esa década un número de egresados/as cercano a los dos mil profesores/as en educación física.

En este periodo, la matriz instruccional del INEF estuvo atravesada y sujetada por el discurso biomédico articulado con ciertas nociones provenientes del campo pedagógico y psicológico. Poco a poco el profesor de educación física se fue convirtiendo en un aplicador/traductor/instructor de ejercicios físicos y actividades lúdicas teniendo en cuenta algunos criterios como la edad y el sexo de los alumnos y, al mismo tiempo, se consolidó una función central legitimadora de su oficio, a partir del avance de materias como antropometría, biometría o biotipología, la de ser el gran medidor corporal: talla, peso, envergadura física, perímetro torácico, cualidades corporales, etétera. La formación de la figura del profesor/a de Educación Física afianzó la transmisión unificada de ciertos saberes (fundamentalmente los provenientes de la anatomía, la mecánica, la antropometría y la fisiología), determinadas prácticas (SAEF) y la metodología más conveniente para alcanzar los objetivos corporales establecidos.

Lentamente el INEF se convirtió en un actor central en la definición de las políticas vinculadas con la "cultura física", especialmente la escolar. En la práctica, en la clase de educación física propiamente dicha, el discurso médico reguló y administró el universo kinético de los alumnos/as dejando sus huellas y marcas en la propia organización pedagógico-didáctica: permitió -o prohibió - distintos tipos de ejercicios físicos (prescribió ejercicios respiratorios, emotivos, sofocantes, higiénicos, descongestionantes, ortopédicos y proscribió los ejercicios congestivos, deformantes, atléticos, acrobáticos, exhibicionistas, hipertróficos y anti-respiratorios), definió los distintos momentos de la clase (tres momentos para clases dirigidas a niños de 6 a 10 años y siete momentos para niños mayores), los momentos de reposo físico (después de un ejercicio de sofocación), la duración total de la clase (ideal: entre 45 y 50 minutos), la cantidad de clases semanales (ideal: tres veces por semana), el momento ideal de dictado de la clase de educación física (al terminar la segunda hora escolar), el cálculo de intensidades de los ejercicios físicos para cada momento, los efectos fisiológicos deseados (primero efectos generales, particulares después y generales al final de la clase), el orden de ejecución de cada ejercicio $\left(1^{\circ}\right.$ Ejercicios preliminares, $2^{\circ}$ Ejercicios de suspensión, $3^{\circ}$ Ejercicios de equilibrio, $4^{\circ}$ Ejercicios del tronco, $5^{\circ}$ Reposo relativo, $6^{\circ}$ Ejercicios sofocantes, $7^{\circ}$ Ejercicios respiratorios), los tiempos de cada ejercicio $\left(1^{\circ}, 2^{\circ}\right.$ y $3^{\circ}$ ejercicios: $3 / 10$ del tiempo total; $4^{\circ}$ y $5^{\circ}$ ejercicios: $2 / 10$ del tiempo total y $6^{\circ}$ y $7^{\circ}$ ejercicios: 5/10 del total), el tipo de alimentación y vestimenta, entre otros tópicos (Scharagrodsky, 2014).

Pero la propuesta "romerista", dominante y hegemónica en las primeras tres décadas del siglo $\mathrm{XX}$ en el ámbito escolar argentino, apoyada por importantes y respetados funcionarios del Consejo Nacional de Educación, destacados y democráticos pedagogos de Estado, gran parte de la comunidad docente (maestros/as) y miembros del INEF, representados por Enrique Romero Brest y su grupo de docentes, encontró tensiones y enfrentamientos por parte de ciertos actores sociales durante las primeras tres décadas del siglo XX — con picos de tensión 
en la primera década, a mediados de los veinte y a principios del treinta - : los herederos de la Sociedad Sportiva Argentina, los responsables de la Dirección General de Tiro y Gimnasia, los funcionarios del Ministerio de Guerra, ciertos políticos y pedagogos tradicionales y conservadores, y los egresados de la otra institución de formación existente en este periodo: la Escuela de Gimnasia y Esgrima del Ejército surgida en 1897. ${ }^{4}$ En estas tensiones lo que se puso en juego fue algo más que un sistema de ejercitación física, determinados tipos de movimientos o ciertos desplazamientos "permitidos", con su gradación, intensidad, seriación y clasificación correspondiente (Levoratti y Scharagrodsky, 2019). La cuestión fue más bien la definición e imposición de concepciones absolutamente diferentes sobre cómo conceptualizar la pedagogía, la política, los cuerpos o la escuela (Scharagrodsky, 2011; Galak, 2012).

Por un lado, el SAEF tuvo como fin formar parte de una escuela republicana y democrática en donde la solidaridad y el respeto a los derechos individuales fuesen la prioridad. Vale decir, el discurso romerista proclamó la libertad individual y una educación para la vida civil, imbuida en el espíritu de solidaridad de una verdadera democracia. Asimismo, la disciplina escolar fue conceptualizada a partir de la razón y en función de principios científicos. La propuesta romerista, con ambivalencias y ciertas contradicciones, alentó un tipo de ciudadanía republicana basada en el respeto a las leyes y a los derechos individuales. En consecuencia, la crítica a la educación física militarizada también implicó un fuerte rechazó a la disciplina de la obediencia y a la jerarquía como elementos vertebradores de las relaciones escolares y sociales. En algún punto, la oposición a la gimnasia militarizada o a los batallones escolares representó la defensa de la autonomía de los niños y el reconocimiento de los derechos del individuo frente al condicionamiento de la libertad individual ante un superior, por el mero hecho de serlo o la subordinación al colectivo "nacional".

Por otro lado, la gimnasia militar escolar, las prácticas de tiro y los batallones escolares, en momentos de tensión bélica y frente a un clima militarista (Bertoni, 2001), tuvieron como fin contribuir a la formación de una escuela tipo cuartel en donde la obediencia, la subordinación, el trato militar y la glorificación de la fuerza fuesen los pilares de la relación pedagógica. La organización social pensada por los defensores de la propuesta militar estuvo basada en la imposición de la fuerza más que en la razón y en la búsqueda de un tipo de organización social atravesada por la jerarquía. Asimismo, las prácticas militaristas difundieron un ideal de patria en donde no prevaleciera el derecho, la libertad individual o la ciencia; sino un ideal guerrero basado en un patriotismo exacerbado y de exclusión de lo 'otro', cuya consecuencia fuese la inevitable lucha entre distintos hombres o naciones.

A pesar de las tensiones y conflictos entre ambas matrices de formación, la propuesta romerista se mantuvo dominante, especialmente, en el escenario escolar en las primeras tres décadas del siglo XX. Sin embargo, este proceso de constantes disputas por monopolizar la educación de los cuerpos se quebró definitivamente a principios de la década de los treinta. La balanza se inclinó definitivamente hacia aquellos maestros de gimnasia y esgrima provenientes del Ejército, de la Dirección General de Tiro y Gimnasia, así como a los grupos de pedagogos, funcionarios escolares y políticos que defendieron esta concepción. En consecuencia, a partir del golpe de 1930, la corporación militar logró expulsar y jubilar de oficio a Romero Brest de la dirección del INEF y apropiarse de las nacientes estructuras estatales de administración y control de los cuerpos, como la primera Dirección General de Educación Física y Cultura o el

\footnotetext{
${ }^{4}$ La Escuela de Gimnasia y Esgrima del Ejército fue creada en 1897 y disuelta en 1903, siendo reabierta 22 años después. Esta institución sobresalió - junto con la Dirección General de Tiro y Gimnasia- porque muchos de sus egresados fueron figuras claves en la promoción y difusión de una educación física militar y se convirtieron en los máximos opositores a la propuesta romerista.
} 
Consejo Nacional de Educación Física. Y fue en esta década cuando, como nunca antes, bajo la reforma educativa bonaerense de Fresco-Noble, se prescribió nuevamente como obligatoria la práctica de tiro en la escuela, se establecieron cursos de formación en educación física para maestros con un tono belicista y se volvió a militarizar dicha especialidad.

\section{La estabilización y consolidación de la matriz instruccional de formación en Educación Física. El INEF y los nuevos actores}

A fines de los treinta, en el marco de gobiernos conservadores y de políticas sanitarias, religiosas y eugenésicas cada vez más presentes desde el Estado y desde ciertos actores sociales (Miranda, 2012; Biernat y Ramacciotti, 2014), se produjo una avanzada de grupos militarizados, ya que los profesores militares egresados de la Escuela de Gimnasia y Tiro del Ejército tuvieron un papel central en diferentes espacios e instituciones (Dirección General de Educación Física y Cultura del país (1936), Consejo Nacional de Educación Física (1937), Dirección General de Educación Física Nacional (1938), etcétera). En este escenario, el INEF se reinventó en 1938 con dos instituciones, aunque mantuvo la autoridad epistémica a la hora de formar docentes en Educación Física.

Concretamente, la formación de las mujeres se mantuvo en la antigua institución de fuerte impronta romerista, pero el 31 de marzo de 1939, el entonces Ministro de Justicia e Instrucción Pública, Jorge Coll, estableció el funcionamiento del nuevo Instituto Nacional de Educación Física "Gral. Manuel Belgrano”. Su característica central fue que acogió sólo a varones, buena parte de ellos becados y provenientes del interior del país, en formato de internado, construyendo lentamente en los estudiantes, a partir de una rutina diaria de estudio y práctica, un conjunto de hábitos, no siempre totalmente aceptados, pero sí definitorios sobre cómo entender al futuro profesor de Educación Física: orden, prolijidad, pulcritud, presencia física, estética viril, obediencia, uniformidad, respeto, uso productivo del tiempo, compañerismo, responsabilidad, colaboración, confianza mutua, sentido de pertenencia a la gran familia del internado, orgullo de pertenecer al INEF, sentido de vocación, fueron algunos de los valores explícitamente señalados por las autoridades, docentes y estudiantes. En muchos sentidos, se priorizó “el 'ser' sobre el 'saber' y la formación práctica sobre la teórica" (Feiguin, 2020: 114).

En este periodo se consolidó el concepto de selección físico-moral de los aptos y de exclusión de los inaptos en la propia experiencia de formación de los estudiantes con pruebas físicas de rendimiento, mediciones antropométricas y aplicación de diversas fichas de revisiones médicas. El discurso médico siguió muy presente a la hora de justificar las intervenciones de un buen docente de la especialidad. Sin embargo, los enfoques pedagógicos y disciplinares incorporaron algunas nociones y categorías diferentes al periodo anterior ya que lentamente surgieron ciertas ideas vinculadas al escolanovismo (difusión del sentido de colaboración y cooperación, estímulo de la vida al aire libre, transmisión de algunos sentidos vinculados con el autogobierno en el internado, cierta retórica sobre la enseñanza basada en el interés de los estudiantes, etcétera) y tuvieron mayor preponderancia otros contenidos como nuevas gimnasias más 'expresivas' (la neosueca, la danesa, la rítmica, con aparatos, etcétera) y una mayor presencia de los deportes en la enseñanza. El registro psicológico (psicología infantil, psicología adolescente, psicología de niños excepcionales, etcétera) comenzó a ganar terreno en la formación docente, aunque siempre articulándose y dependiendo de los saberes biomédicos y, en menor medida, pedagógicos. La irrupción del bautismo, la entrega de la "I" y de las inter-tribus ${ }^{5}$ como rituales institucionales

${ }^{5}$ El Bautismo fue un acto ritual (pruebas físicas, desafíos corporales, retos simbólicos, etcétera) desarrollado durante buena parte del siglo XX en el cual los estudiantes de primer año del INEF debían atravesar, experimentar y transitar para ser reconocidos como 
de formación vinculados con la transformación e identificación de los estudiantes con la institución, fabricaron una dinámica particular sobre la experiencia educativa fomentado y naturalizando, en muchos casos, prácticas violentas, discriminatorias y estigmatizantes.

Lentamente a partir de 1939, los egresados del nuevo Instituto Nacional de Educación Física "Gral. Manuel Belgrano" hegemonizaron buena parte de los puestos de dirección de organismos estatales, ${ }^{6}$ colegios nacionales, escuelas primarias y clubes particulares en distintas jurisdicciones del país. Fue clave que muchos de los egresados proviniesen de distintas provincias a las cuales regresaban luego de concluir sus estudios. El largo brazo del INEF comenzó a tener influencia más allá de la capital argentina formando líderes provinciales y nacionales ${ }^{7}$ en el campo de la Educación Física. También monopolizaron las primeras jornadas y congresos sobre Educación Física, los cuales fueron organizados en ese periodo por egresados y egresadas de ambos INEF. ${ }^{8}$ La separación por sexos de estas dos instituciones se mantuvo hasta 1967, año en que se integraron ambos sexos y se unificó el INEF.

En términos generales, en este periodo la formación del INEF "Gral. Manuel Belgrano" estuvo atravesada por "aportes del movimiento escolanovista”, articulados con la influencia religiosa (presencia de vínculos con la Iglesia) y moral de la "Asociación Cristiana de Jóvenes" y por una persistencia recontextualizada de la "matriz militar (competencias de tiro en el INEF, vacantes para profesores egresados del INEF en el Cuerpo de Gimnasia y Esgrima del Ejército, cierta lógica pedagógico-didáctica castrense en el interior del INEF, etcétera)" (Feiguin, 2020: 115).

Ambos INEF (la sección varones y la sección mujeres), en los años cuarenta y cincuenta mantuvieron la hegemonía a la hora de producir docentes en Educación Física, definiendo un ideal de formación a partir de planes y programas de estudio específicos, seleccionando ciertos contenidos (nuevos tipos de gimnasia y una mayor presencia de los deportes), prescribiendo determinada bibliografía (además de la francesa, se introdujo con fuerza la bibliografía norteamericana y la alemana), estableciendo formas de ingreso y exámenes más exigentes (se intensificaron las pruebas de ingreso y selección de los más o menos aptos), estableciendo rituales (se consolidó el bautismo y las inter-tribus), fomentando tipos de titulaciones (se diversificaron las titulaciones, por ejemplo, surgió el "Maestros de Gimnasia y Recreación”), generando nuevas formas de inserción laboral (además del aumento de instituciones educativas formales, se incrementaron las inserciones laborales en clubes, en nuevas reparticiones estatales y en experiencias educativas no formales como, por ejemplo, las colonias de vacaciones, difundidas en muchas provincias) y fabricando una particular cultura institucional (el internado en los varones).

integrantes orgullosos de dicha institución. Sin reglamentaciones institucionales específicas (curriculum oculto), la organización del bautismo estuvo, en general, bajo la responsabilidad exclusiva de los alumnos mayores, los cuales difundieron a través del Bautismo ciertos valores como el respeto a la autoridad, el sometimiento a las jerarquías, la obediencia a los mayores y el aprendizaje de determinados códigos morales como la responsabilidad, la puntualidad, la solidaridad, la cooperación, la importancia de la apariencia y el honor. En muchos casos, en nombre de esta tradición se legitimaron comportamientos violentos y humillantes. Una vez pasado el Bautismo, se entregaba la I y los ingresantes se convertían en integrantes de "la gran familia" del INEF conformando una tribu (grupo de estudiantes) para competir y compartir momentos formativos en la institución.

${ }^{6}$ Entre los años cuarenta y cincuenta se crearon una gran cantidad de direcciones de Educación Física provinciales con participación de egresados del INEF “Gral. Manuel Belgrano": Salta (1944), Mendoza (1944), Jujuy (1948), Santiago del Estero (1950), Tucumán (1950), Entre Ríos (1951), La Rioja (1952), Corrientes (1953), San Juan (1955), etcétera (Erdociaín, 2019: 97-98).

7 'Adalides' deportivos fue el término utilizado por las autoridades del INEF y por los políticos que frecuentaron el INEF, entre ellos ministros y presidentes, para referirse a los egresados y su importante misión nacional.

${ }^{8}$ En este periodo, la APEF (Asociación de Profesores de Educación Física) organizó varios congresos y jornadas: 1942, 1944, 1947, 1950, 1956, entre otros. Entre los más destacados se puede mencionar la Primera Reunión Sudamericana de Asociaciones de Profesores de Educación Física, realizada en 1950. 
Más allá de algunas tensiones internas (Beer, 2014), la gran familia homosocial del San Fernando, y en menor medida el INEF de mujeres, se convirtieron en el universo de referencia a la hora de formar profesores en Educación Física en este periodo.

Ciertamente, en la primera mitad del siglo XX sólo cinco instituciones gobernaron la formación del oficio de 'educador físico' en Argentina. Una militar, que funcionó en forma intermitente, aunque mantuvo importantes cuotas de poder en ciertos momentos y espacios (1897-1902, reabierta en 1925-1934) y cuatro instituciones civiles. De estas últimas, los dos INEF ya mencionados (uno creado en 1901 y otro en 1938 sólo para varones) fueron los "verdaderos" productores de discursos sobre el significado del buen docente en Educación Física. A mediados de los cuarenta otras dos instituciones, con menor capacidad de influencia sobre el campo de la cultura física y las políticas deportivas, fueron creadas en Argentina. El IPEF de Córdoba, fundado en 1946 (García, 2006: 281) y la Escuela de Educación Física de Rosario, que abrió sus puertas en 1944. En ambos casos, hubo presencia de docentes egresados de los INEF de la capital argentina (Cena, García y Tulisse, 2012), los cuales instalaron y reforzaron lógicas, saberes, dinámicas y rituales producidos en la institución modelo.

Cabe destacar que en los años cincuenta las lógicas macro-políticas tuvieron algunos efectos en el INEF San Fernando. Esta institución, que hegemonizó la formación de profesores en la especialidad, incorporó algunos cambios vinculados al primer peronismo y su fuerte relación con las políticas deportivas, especialmente hacia actores sociales hasta esos momentos excluidos de ciertas experiencias corporales (Orbuch, 2016) y, al mismo tiempo, padeció la intervención política a la institución producida luego del derrocamiento de Perón en 1955.

Por último, en esa década, al campo de la formación de docentes en Educación Física se sumaron dos instituciones universitarias: la Universidad Nacional de Tucumán, con antecedentes previos (González de Álvarez, 2006) y la Universidad Nacional de La Plata (UNLP). Esta última, a partir del Profesorado Universitario de Educación Física (PUEF), consolidó muy lentamente una matriz de formación diferente a los INEF en lo que respecta a planes y programas de estudio y cultura institucional (más ligada al funcionamiento de una universidad y no de un internado o institución terciaria), transmitiendo contenidos y bibliografía vinculados con una posición menos instrumental de la enseñanza que los INEF y más pedagógico-humanista. Como señala Villa (2007: 128) "la formación no estaría circunscripta solamente a aspectos técnicos y prácticos de la gimnasia y el deporte sino que se complementaría con una formación de corte teórico contextual sobre aquellos y una formación humanística y pedagógica general que impartiría la Facultad de Humanidades". Muy lentamente, en la segunda parte del siglo XX, el PUEF se convirtió para los INEF en la 'otredad' a combatir a la hora de pensar en un ideal de "buen" profesor en Educación Física. La forma de denigrar y cuestionar la formación universitaria fue a partir de la siguiente frase: "los egresados del PUEF son muy teóricos y no saben nada de la práctica concreta, de la clase". Pero la otredad fue especular, ya que para varios referentes del PUEF, en el INEF "sólo enseñaban cuestiones prácticas, puramente técnicas y sin sustento teórico". En ésta disputa - en parte 'real' y en parte imaginaria- durante este periodo el INEF mantuvo la hegemonía discursiva y práctica, y su capacidad de influencia en las políticas educativas y deportivas nacionales y provinciales.

\section{La multiplicación de las matrices de formación en la Educación Física: el INEF como modelo instituyente se propaga a nivel nacional}

A partir de la década de los sesenta y, muy especialmente en los setenta y principios de los ochenta, los INEF se multiplicaron y se expandieron en varias 
regiones del país (Levoratti, 2015). No sólo los docentes formados en el INEF llegaron a ciudades y pueblos del interior del país, sino que en este periodo se crearon, por primera vez, nuevas instituciones de formación en Educación Física en varias provincias argentinas siguiendo el modelo del INEF capitalino.

Por ejemplo, en 1960 comenzó a funcionar el Instituto Nacional de Educación Física de Santa Fe (Galantini, 2000). En 1962 se creó el INEF en la provincia de Mendoza. A fines de los sesenta, y a partir de cambios en la legislación y en las políticas educativas, nuevos profesorados de educación física se constituyeron: en 1968 en la provincia de Río Negro; en 1970 en la provincia de Corrientes; en 1971 en la provincia de Buenos Aires; en 1979 en la provincia de Jujuy con importantes antecedentes previos (Toconás, 2006), y en 1980 en la provincia de Formosa. Todas estas instituciones tuvieron un carácter estatal, terciario, público y fueron de acceso gratuito. En 1974 se creó la tercera propuesta universitaria de formación en educación física en el país, en la Universidad Nacional de Río Guarto, Córdoba. Ese mismo año, comenzaron a instalarse propuestas terciarias de formación de la especialidad de forma privada. También en esa década se creó el primer profesorado de educación física de la provincia de Buenos Aires (1971), apoyado por el Estado y por empresas privadas (Fundación Fortabat), y otros institutos en ciudades bonaerenses, por ejemplo, Bahía Blanca, Lomas de Zamora, Mar del Plata, General las Heras y Pehuajó en 1979. "Entrando en la década del ochenta, se realizó la última ola de aperturas de INEF, en 1982 se abrieron en las provincias de Catamarca, San Juan, Entre Ríos y en 1983 en Chubut" (Levoratti, 2015). Este proceso fue acompañado en los primeros años de la década de los ochenta por la creación de una gran cantidad de Centros de Educación Física $(\mathrm{CEF})^{9}$ con una fuerte inserción y presencia de egresados y egresadas de los INEF.
Cabe mencionar que, durante la dictadura cívico-militar (1976-1983) el INEF de capital ya unificado (varones y mujeres), re-significó "junto a sus tradiciones institucionales, relaciones y prácticas pedagógicas pre-existentes, convergiendo en un conjunto híbrido que desplegó una perspectiva técnica y operativa. En ella se fue profundizando la deportivización del Instituto y de la Educación Física, en un entorno profundamente disciplinario y de control corporal" (Beer, 2014: 367). En esos años muchas voces disidentes de las instituciones de formación de la especialidad sufrieron el terrorismo de Estado, la violencia política, la censura, la persecución ideológica y tuvieron docentes y estudiantes desaparecidos.

En cualquier caso, un dato es muy significativo: la amplia mayoría de las instituciones de formación creadas a lo largo de este periodo tuvieron una importante cantidad de docentes provenientes de los INEF de la capital argentina. De igual manera, la emergente producción, traducción y circulación de libros, bibliografía y material específico para la especialidad, fundamentalmente a través de las editoriales Stadium, Paidós y, en menor medida, Kapelusz, fue monopolizada centralmente por egresados del INEF. Estos últimos se convirtieron en muchas ciudades y pueblos del interior en los verdaderos 'adalides' o líderes del deporte a partir de sus inserciones laborales: instituciones educativas (como docentes e inspectores), clubes, reparticiones estatales, etcétera. Asimismo, las noveles instituciones se nutrieron, con ciertos reparos, matices y traducciones locales, de la matriz instruccional y de los rituales, normas, simbologías, lógicas, estéticas, vestimentas y sentidos de la institución "modelo".

Sin embargo, hubo varios cambios de la matriz instruccional con relación al periodo anterior. Entre los años sesenta y setenta, lentamente, en las razones fisiológicas de los ejercicios físicos, el organismo como ser susceptible de perfección y modificación

${ }^{9}$ Entre 1980 y 1983 se crearon CEF en Santa Fe, Entre Ríos, Corrientes, Tucumán, Buenos Aires, Catamarca, Córdoba, Chubut, Santa Cruz, etcetera. 
física, las condiciones fisiológicas de la clase, la gradación muscular en los ejercicios, los criterios médicos para realizar correctamente un ejercicio, la medición de la salud, la medición de la fuerza y de otras aptitudes especiales; se empieza a mencionar el respeto por la maduración del niño, el necesario conocimiento de la evolución psicológica de la infancia, el desarrollo de diferentes niveles de aprendizaje anclados en la psicología evolutiva, la motivación de los niños, las diferencias individuales o la psicogénesis del niño como condición para adaptar los juegos y las prácticas deportivas. Un proceso que se inició muy tenuemente en los entre 1920 y 1930 , pero se consolidó en los sesenta. Es decir, esto último se visibilizó claramente en los años sesenta y setenta. Se pasa en los planes, programas y manuales de la especialidad de Demeny, Tissie, Mosso y, muy especialmente, Lagrange, a Gesell, Piaget, Erikson o Wallon. El desplazamiento fue bastante sutil ya que los "representantes psicológicos" siguieron atados a registros provenientes de la biomedicina (Scharagrodsky, 2004).

Por otra parte, desde el registro pedagógico, especialmente en las décadas de los sesentas y los setentas, fue hegemonizado por la obra de Annemarie Seybold, fiel representante de la escuela de Colonia, Alemania, ${ }^{10}$ la cual apareció profusamente citada en los planes de formación para profesores y maestros en educación física, así como en la bibliografía de muchos de los planes escolares, y en la mayoría de los manuales y textos escolares de educación física. ${ }^{11}$

La matriz instruccional de los INEF, pero también de instituciones de formación universitaria, apelaron a nuevos principios provenientes del campo de la pedagogía crítica o renovada. Los libros Principios
Pedagógicos en la Educación Física y Principios Didácticos de la Educación Física escritos por Annemarie Seybold, se convirtieron en material de referencia en el periodo de los sesenta y setenta. Varias ediciones fueron publicadas apareciendo como bibliografía básica y obligatoria en los planes oficiales de enseñanza escolar de 1972 y 1981. La circulación de este material fue tan decisiva en esta disciplina, que muchos profesores de educación física lo consideraron "el catecismo de la formación docente".

Múltiples principios, como el de adecuación al niño, el de individuación, el de solidaridad, el de totalidad, el de intuición y objetivación, el de experiencia práctica o realismo, el axiológico, el de espontaneidad y el de adecuación estructural delimitaron, junto con el saber proveniente de la psicología educacional, qué podía y debía hacer un alumno y una alumna en las clases de educación física. Estos principios comenzaron a erosionar varias de las tradiciones sobre cómo debía enseñar, ser y parecer un buen profesor de Educación Física.

En menor medida Liselott Diem ${ }^{12}$ se convirtió en una referente no tanto de la pedagogía como de la didáctica de la educación física (Scharagrodsky, 2004), realizando en el periodo más de 10 viajes al país, donde dictó conferencias y cursos principalmente de "Educación física infantil" y participó en las instancias de debate y concreción curricular del plan de estudio de los institutos nacionales de Educación Fisca implementados desde 1980 y en el de la licenciatura de la UNLP de 1982. La década del sesenta "fue pródiga en intentos de aplicación directa de los conocimientos psicológicos a la práctica educativa. Las teorías que dominaban el campo psicológico (en especial, la Psicología Genética) fueron

\footnotetext{
${ }^{10}$ A principios de los años sesenta más de 120 docentes en Educación Física participaron en cursos de perfeccionamiento en el exterior, siendo Alemania uno de los países con mayor circulación de egresados de los INEF.

${ }^{11}$ Durante este periodo se intensifico la participación de actores locales en asociaciones y organismos internacionales. Entre ellos se puede nombrar la tarea del licenciado Mario López que fue representante argentino de la International Council for Health, Physical Education and Recreation (ICHPER) y de la International Association of Higher Schools of Physical Education (AIESEP).

${ }^{12}$ En 1961 Carl y Liselott Diem, destacados docentes alemanes, dictaron numerosos cursos y conferencias sobre distintos tópicos referidos a la Educación Física.
} 
tomadas como fuente de información a utilizar de manera directa en la definición de formas de intervención escolar. La psicología fue utilizada para definir objetivos educativos, para diagnosticar niveles de sujetos, para secuenciar contenidos, para estipular una metodología" (Terigi, 2000: 45) y también para habilitar las competencias necesarias para fabricar un buen docente en Educación Física.

Con la restauración de la democracia las matrices de formación comenzaron un lento proceso de revisión de sus planes, programas, contenidos y bibliografía. En 1983 se creó el Instituto Nacional de Tiempo Libre y Recreación y en 1986 se produjo un intensó proceso de debate sobre los planes de estudio destinados a la formación de profesores de educación física de los INEF. En esta instancia se aprobó, por resolución 169 de 1989, una nueva curricula, donde se hacía foco en el carácter pedagógico de la educación física y su accionar sobre la motricidad humana. Aunque el mismo no fue implementado de modo compulsivo en todos los INEF, su aplicación se concretó en los institutos situados en Mendoza, San Juan y Comodoro Rivadavia-Chubut.

Realizando un balance del periodo podemos identificar, a inicios de 1980, un total de 33 instituciones donde se dictaba el profesorado de Educación Física, de las cuales tres pertenecían al sistema universitario y 30 a la educación superior no universitaria. Asimismo, el 62\% de las instituciones eran de carácter público y el restante $38 \%$ privadas.

\section{El crecimiento exponencial de los centros de formación en Educación Física: nuevas voces, sentidos y competencias}

Con la restauración democrática, nuevas perspectivas y políticas educativas emergieron y la formación en Educación Física no estuvo exenta de todo ello. Desde mediados de los ochenta - momento en que se crearon los institutos de Avellaneda en la Provincia de Buenos Aires y el del Parque Chacabuco en la Capital Federal-, con mayor lentitud y aceptación que otras disciplinas educativas, comenzaron a surgir algunos cambios en las instituciones de formación en Educación Física. Hubo modificaciones en las matrices de formación, en los planes y programas de estudio, en ciertos contenidos y una renovación de la bibliografía, cuestionando ciertas tradiciones fuertemente arraigadas en la formación de la especialidad, como el ingreso restrictivo, los exámenes físicos y de rendimiento, los rituales típicos y distintivos (Bautismo, inter-tribus, etcétera), la separación por sexos en la enseñanza de ciertos deportes, la formación fuertemente biologicista, el deportivismo a ultranza, la descontextualización del oficio, etcétera. También, en algunas pocas instituciones de formación, hubo sumarios a aquellos que colaboraron con la dictadura cívico-militar. Asimismo, surgieron nuevas formas de inserción laboral que potenciaron la matriz instruccional con contenidos y tópicos hasta esos momentos no enseñados como, por ejemplo, la enseñanza del circo, la problematización y contextualización de los juegos nativos, la introducción de ciertos bailes y danzas o la incorporación deportes alternativos.

En términos cuantitativos, "sí hacemos un recuento en el año 1987 se identifican 54 establecimientos educativos en los cuales se desenvolvía la carrera. El 86.4\% de ellos eran, en términos de hoy en día, instituciones de educación superior no universitarias y el restante $13.6 \%$ de carácter universitario. Asimismo, el $60.5 \%$ de las propuestas eran de gestión pública y el 39.5\% privada" (Levoratti, 2015).

En los años noventa como se puede observar en el gráfico 1, se multiplicaron año con año las propuestas educativas radicadas en instituciones terciarias no universitarias privadas, muchas de ellas interesadas en el lucro y con una débil base epistemológica vinculada a la enseñanza. Este tipo de oferta formativa comenzó a incrementarse en los primeros años posteriores a la recuperación de la democracia, teniendo un primer pico de incremento en los inicios de los años noventa y un segundo pico hacia fines de la 
década y los primeros años del nuevo siglo. Asimismo, durante los años noventa solamente se abrió el profesorado de educación física en dos instituciones de carácter público. Hubo que esperar hasta 2002 y con mayor intensidad el 2008 para identificar un incremento sostenido de ofertas formativas de dicho carácter.

Gráfico 1. Instituciones que iniciaron el dictado de la carrera de profesorado de Educación Física por año y modalidad de inscripción dentro del sistema de educación superior 1912-2020

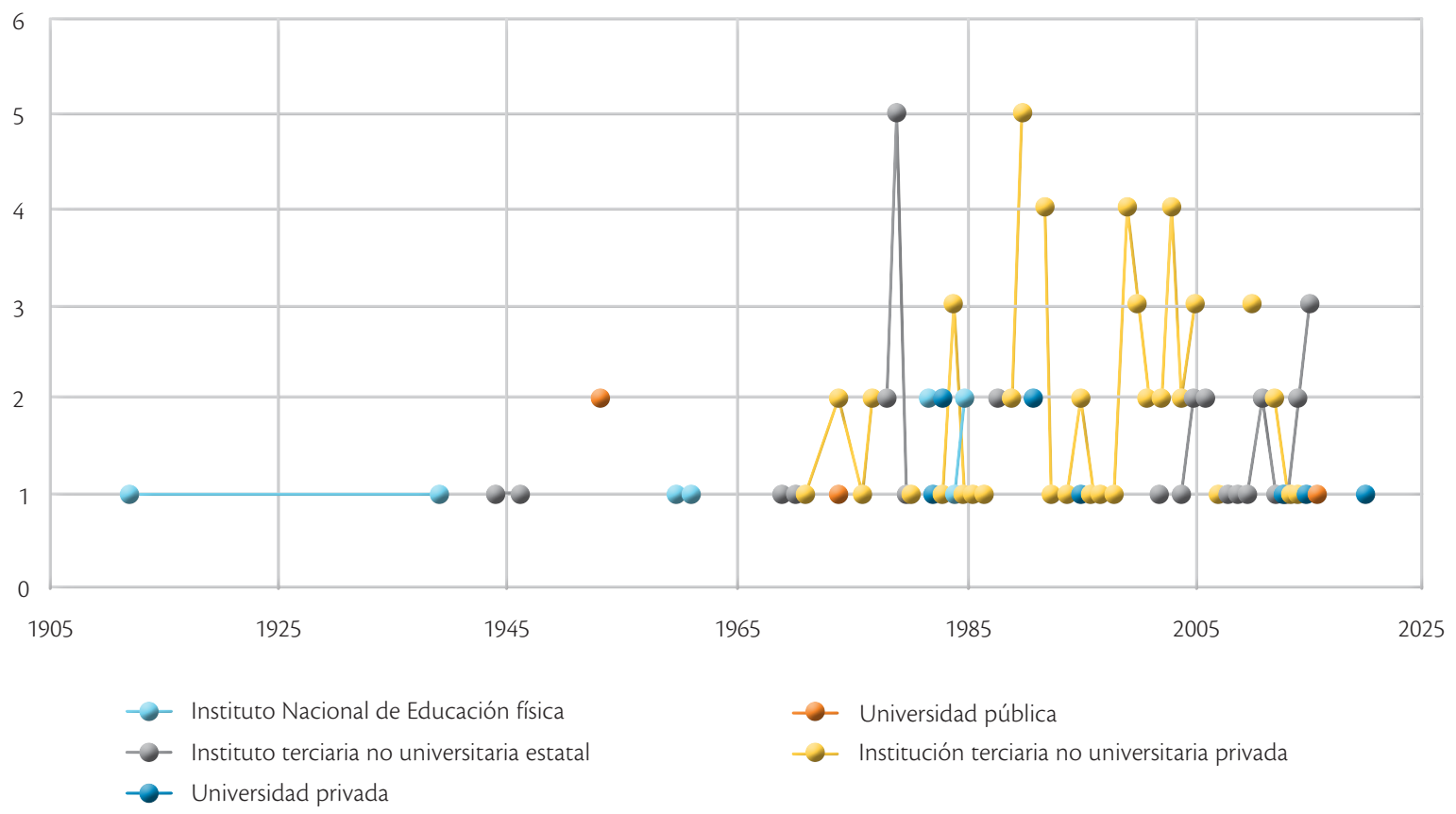

Fuente: elaboración propia con base en el relevamiento de datos realizado en 2020 en las instituciones que dictaban el profesorado de educación física en Argentina.

Tabla 1. Cantidad de instituciones de acuerdo a su inscripción dentro del Sistema de Educación Superior, 2020

\begin{tabular}{|c|l|c|c|}
\hline Tipo de institución & \multicolumn{1}{|c|}{ Modalidad de gestión } & Porcentaje & Cantidad \\
\hline \multirow{2}{*}{ Universidad } & Pública & $5.90 \%$ & 11 \\
\cline { 2 - 4 } & Privada & $5.90 \%$ & 11 \\
\hline \multirow{3}{*}{ Institución no universitaria } & Pública & $37.3 \%$ & 72 \\
\cline { 2 - 4 } & Privada & $51.3 \%$ & 99 \\
\hline
\end{tabular}

Fuente: elaboración propia en base al relevamiento de datos realizado en el año 2020 en las instituciones que dictaban el profesorado de educación física en Argentina. 
Cuantificando la cantidad de instituciones donde se dicta el profesorado de educación física en 2020, advertimos que estas últimas dos décadas modificaron sustancialmente el cuadro de situación. De ser una oferta educativa desarrollada preponderantemente en institucionales estatales tanto de carácter nacional como provincial, nos encontramos en 2020 que el $57 \%$ se dicta en establecimientos privados. Asimismo, en la tabla 1 se puede observar la preponderancia de las ofertas radicadas en dependencias que pertenecen al sistema de educación terciario no universitario, concentrando el 88\%.

En los últimos 20 años el crecimiento de las instituciones de formación fue exponencial. Se sumaron nuevas instituciones a la formación de profesores de la especialidad pero bajo un régimen universitario, entre ellas podemos mencionar las universidades Nacional de La Matanza, Luján, Comahue, Hurlingham, José C. Paz, La Rioja, Provincial del Córdoba, Autónoma de Entre Ríos y en las universidades privadas la Universidad Católica de La Plata, Instituto Universitario de la YMCA, Instituto Universitario River Plate, Universidad Metropolitana para la Educación y el Trabajo, Universidad de Flores, Universidad Abierta Interamericana, Universidad Adventista del Plata, Universidad de Concepción del Uruguay, Universidad "Juan Agustín Maza", Universidad Gastón Dachary y la Universidad Católica de Salta. Además, debemos considerar la realización de ciclos de complementación curricular para la obtención de títulos de licenciado en universidades nacionales, como fue el caso de las universidades Nacional de La Plata, Nacional de Lanús, Nacional de Lomas de Zamora, Nacional de Lujan, Nacional de San Martín, Nacional del Oeste, Nacional Noroeste de la Provincia de Buenos Aires, Provincial del Sudoeste, Nacional de Catamarca, Nacional del Chaco Austral, Nacional de Villa María, Provincial de Córdoba, Autónoma de Entre Ríos, Nacional de La Rioja, Nacional de Río Negro, Nacional de Villa Mercedes, Nacional de Rosario, Nacional del Litoral y Universidad Nacional de Tucumán. Esta formación también se dictó en instituciones privadas, como es el caso de la Universidad Fasta, Universidad Blas Pascal, Instituto Universitario de la YMCA, Instituto Universitario River Plate, Universidad de Flores, Universidad Abierta Interamericana, Universidad Adventista del Plata, Universidad Católica Argentina -Sede Mendoza, Universidad 'Juan Agustín Maza', Universidad Católica de Cuyo- Sede San Juan, Universidad del Aconcagua, Universidad Gastón Dachary, Universidad Católica de Salta, Universidad de Concepción del Uruguay y Universidad Católica de Santiago del Estero.

A partir de la reforma educativa de $1993^{13}$ los institutos nacionales fueron transferidos a las administraciones provinciales o a universidades. Este cambio de dependencia implicó que cada jurisdicción tuviera que confeccionar sus diseños curriculares. Sumado a ello, desde principios de los años noventa en la Argentina se formaron espacios de discusión sobre la identidad disciplinar y se impulsaron trayectos formativos complementarios de licenciatura, instancias que promovieron el acercamiento hacia algunos debates provenientes de las ciencias sociales y de la educación. Todo ello potenció una mirada más crítica y contextualizada sobre la matriz formativa de los INEF. Al mismo tiempo, estos enfoques posibilitaron legitimar a algunos actores y por transferencia a la disciplina en las instituciones universitarias y en las instancias de definición curricular.

A partir de los noventa, el PUEF desplazó a los INEF muy lentamente en el monopolio de la producción, distribución y transmisión de saberes sobre la educación física. El nuevo modelo de relación entre el Estado y la universidad en los noventa, caracterizado como un "Estado evaluador" interesado en la

\footnotetext{
${ }^{13}$ En la reforma educativa de los noventa, hay participación de docentes del PUEF.
} 
producción académica, así como la pro-actividad de los agentes de la especialidad (Felipe, 2019), afianzaron el prestigio del PUEF dentro y fuera de la universidad. Sus egresados y egresadas comenzaron a disputar espacios en instituciones educativas, en direcciones provinciales y nacionales de educación física y de deportes, y a producir literatura específica con alta circulación e impacto - nacional y regional- y con una lógica académica. ${ }^{14}$ La matriz de formación se nutrió de literatura actualizada proveniente de las ciencias sociales y humanas con una visión histórica, contextual y relacional de la Educación Física y del futuro docente de la especialidad (Levoratti, 2018; Ron, 2019).

Si hasta los años sesenta y setenta la potestad sobre la formación docente en Educación Física fue monopolizada por la matriz de los INEF, lentamente a partir de los años ochenta y, muy especialmente, a partir de los noventa, la situación cambió ya que se modificaron las relaciones de fuerza entre las diversas instituciones, la visibilidad y el poder de influencia de las mismas. Sin duda, las instituciones universitarias que surgieron en los últimos 20 años modificaron las posiciones en el campo sobre quiénes tenían el derecho a hablar y con qué autoridad en la Educación Física y en la formación docente de la especialidad.

\section{Conclusiones}

Los procesos de institucionalización de formación del oficio de Educación Física son producto de una compleja, variada y rica historia. A lo largo del siglo $\mathrm{XX}$ hemos identificado cuatro etapas distintas.

Un primer periodo fue el momento en que se inventó una determinada tradición (la romerista) sobre el oficio de enseñante en la Educación Física en las primeras tres décadas y media del siglo XX abarcando el periodo comprendido entre 1901 a 1938. Un segundo periodo fue producto de la estabilización y consolidación de la matriz instruccional, con cambios y continuidades en la formación en Educación Física. El INEF (sección varones y mujeres) se afianzó como dispositivo de formación monopólico, con impacto nacional entre fines de los años treinta hasta principios de los años sesenta. En este periodo se produjo la emergencia de unas pocas instituciones de formación en la especialidad. Un tercer periodo, entre los años sesenta, setenta y principios de los ochenta, se generó a partir de la propagación de las matrices de formación en la Educación Física en varias ciudades y provincias argentinas siguiendo, con matices, el modelo del INEF de los periodos anteriores, aunque incorporando nuevas perspectivas pedagógicas, didácticas, psicológicas y disciplinares. Recién en este periodo se inició la federalización de la formación, aunque con una fuerte injerencia del Estado nacional, ya que estuvo presente de modo directo al ser sus propuestas curriculares las que se implementaron en los institutos nacionales y en los establecimientos de educación privada, y de forma indirecta por ser considerado por los docentes como el modelo legítimo a ser replicado/traducido en las instituciones provinciales. Este periodo consolidó una serie de rituales, prácticas, lógicas y sentidos, aunque, al mismo tiempo, los mismos fueron resignificados regionalmente por los actores y las tensiones locales y provinciales.

Por último, el cuarto periodo aconteció a partir de mediados de los años ochenta y, muy especialmente, en la década de los noventa, en el marco de importantes cambios en las políticas educativas, con nuevos marcos epistémicos y regulatorios. Estos generaron

\footnotetext{
${ }^{13}$ Esto fue, en parte, gracias al Programa Nacional de Incentivos a la Investigación iniciado en todas las universidades en los años noventa, a las becas e intercambios universitarios, a las lógicas universitarias vinculadas con las publicaciones, al ingreso al CONICET y a la CIC de parte de algunos docentes del PUEF, a la categorización de docentes del PUEF, a la edición de revistas académicas, y a la organización de jornadas y congresos de la especialidad, entre las que se destacó el Primer Congreso de Educación Física y Ciencias iniciado en 1993, el cual aún continua.
} 
nuevos sentidos en la matriz instruccional en algunos centros de formación y un aumento exponencial de los mismos en términos generales, pasando de ser aproximadamente 30 a principios de los ochenta a 193 en la actualidad, la mayoría pertenecientes al sistema de educación superior no universitario. En este periodo la matriz de formación de los INEF comenzó a ser desplazada en términos de poder, reconocimiento académico, prestigio y autoridad por otras instituciones de formación, especialmente las universitarias, destacándose el PUEF de la UNLP con su protagonismo como centro productor y difusor de saberes específicos, con una sustantiva producción académica y con una cada vez mayor influencia de sus egresados y docentes en espacios de formación y en la definición de ciertas políticas vinculados al campo. Asimismo, en este periodo se visibilizó como nunca antes la creación de muchas instituciones

\section{Referencias}

Aisenstein, Ángela (2007), "La matriz disciplinar de la educación física. Su relación con la escuela y la cultura en el contexto nacional (Argentina 1880-1960)", en Carmen Soares (org.), Pesquisas sobre o corpo. Ciências humanas $e$ educação, Sao Paulo, Autores Asociados, pp. 23-47.

Alliaud, Andrea (2007), Los maestros y su historia: los orígenes del magisterio argentino, Buenos Aires, Granica.

Beer, David (2014), "La configuración de las tradiciones del Instituto Nacional de Educación Física de Buenos Aires y su resignificación en el contexto de la última Dictadura Militar", Tesis de doctorado, Buenos Aires, Flacso, <https://repositorio.flacsoandes.edu.ec/ bitstream/10469/6395/2/TFLACSO-2014DB.pdf>, [Consulta: abril de 2020].

Bertoni, Lilia (2001), "Soldados, gimnastas y escolares: defender la nación", en Patriotas, cosmopolitas y nacionalistas. La construcción de la nacionalidad argentina privadas, varias de ellas con sólo fines de lucro, pasando a representar el $57 \%$ de las propuestas de formación y una intensa competencia entre las distintas instituciones de formación terciaria y universitaria que se constituyeron en los últimos 20 años.

Como pudimos ver en este recorrido de más de 100 años sobre la formación de los profesores de educación física en Argentina, ella se encontró en permanente debate, identificando por más de 80 años un modelo institucional que se conformó como el instituyente. En las últimas tres décadas la formación de grado inicial fue objeto de cambios tanto en términos cuantitativos, por el incremento exponencial de la cantidad de instituciones, como cualitativos, de acuerdo con la inserción de las ofertas educativas dentro del sistema de educación superior, los modelos institucionales en puja y las perspectivas conceptuales disciplinares promovidas.

a fines del siglo XIX, Buenos Aires, Fondo de Cultura Económico, pp. 213-254.

Biernat, Carolina y Karina Ramacciotti (org.) (2014), Historia de la saludy la enfermedad, Buenos Aires, Biblos.

Cena, Marcela, Antonio García y Liliana Tulisse (2012), De nombres propios, lugares e identidades. Tres miradas sobre la historia del Instituto Provincial de Educación Física, IPEF. 1946-2012, Córdoba, Corintios.

Cornejo, Miguel. Carlos Matus y Camilo Vargas (2011), "La EF en Chile: una aproximación histórica", Revista Digital EFdeportes, vol. 16, núm. 161, Buenos Aires, http:/ / www.efdeportes.com/efd161/la-educacionfisica-en-chile.htm [Consulta: marzo de 2020].

Erdociaín, Luis (2019), Procesos de institucionalización y organización de la Educación Física en la estructura del Estado Nacional Argentino (1938-1999), Buenos Aires, Programa Interuniversitario de Doctorado en Educación, 


\section{UNTREF-UNLA-UNSAM.}

Feiguin, Andrea (2020), "El INEF General Belgrano de San Fernando y la consolidación de una cultura escolar para la formación docente (1938-1954/55)", Tesis de Maestría en Ciencias Sociales con Mención en Historia Social, Buenos Aires, Universidad Nacional de Luján.

Felipe, Celeste (2019), Políticas académicas y estrategias de legitimación disciplinar de la Educación Física en la Universidad Nacional de La Plata (1992-2004), Buenos Aires, Maestría en Educación, Universidad Nacional de La Plata.

Galak, Eduardo (2012), "Del dicho al hecho (y viceversa): el largo trecho de la construcción del campo de la formación profesional de la Educación Física en Argentina", Tesis de doctorado, Buenos Aires, Universidad Nacional de La Plata.

Galantini, Guillermo (2000), "Conciencia, tiempo y conocimiento en la institucionalización del INEF/ISEF 'César Vázquez' de Santa Fe”, Revista Digital EFdeportes, año 5, núm. 24, <https://www.efdeportes.com/efd24b/isf. htm>, [Consulta: abril de 2020].

García, Antonio (2006), “Creación del IPEF de Córdoba. Antecedentes, apertura y primeros años de funcionamiento", en Rodolfo Rozengardt (ed.), Apuntes de Historia para profesores de Educación Física, Buenos Aires, Miño y Dávila, pp. 281-316.

González de Álvarez, María Laura (2006), "Departamento de Educación Física de la Universidad Nacional de Tucumán. Un proyecto innovador del año ' 47 ', en Rodolfo Rozengardt (ed.), Apuntes de Historia para profesores de Educación Física, Buenos Aires, Miño y Dávila, pp. 317-336.

González de Álvarez, María Laura (comp.) (2012), La educación fisica en Latinoamérica. Orígenes y trayectorias de la formación docente, Tucumán, EDUNT.

Hobsbawm, Eric (1985), "Introduction: inventing traditions", en Eric Hobsbawm y Terence Ranger (eds.), The Invention of Tradition, Cambridge, Cambrdige University Press, pp. 1.

Levoratti, Alejo (2015), "La creación de los profesorados de educación física en Argentina. 1912-2014", $11^{\circ}$ Congreso Argentino de Educación Física y Ciencias, 28 de septiembre al 10 octubre de 2015, UNLP, Ensenada, Argentina, en Memoria Académica, <http://www. memoria.fahce.unlp.edu.ar/trab_eventos/ev.7372/ ev.7372.pdf> [Consulta: abril de 2020].

Levoratti, Alejo (2018), "Los debates de la educación física y los enfoques de las ciencias sociales. Un análisis de los lineamientos curriculares nacionales destinados a la formación docente en Argentina. (1993-2015)", The Fournal of the Latin American Socio-cultural Studies of Sport, Brasil, vol. 9, núm. 1, pp. 45-63.

Levoratti, Alejo y Pablo Scharagrodsky (2019), "La formación de los educadores físicos civiles y militares en los primeros años del siglo XX en Argentina", Revista Educación Física y Deporte, vol. 37, núm. 1, DOI: https:// doi.org/10.17533/udea.efyd.v37n1a03, [Consulta: marzo de 2020].

Lionetti, Lucía (2007), La misión política de la escuela pública. Formar a los ciudadanos de la república (1870-1916), Buenos Aires, Miño y Dávila.

Ljunggren, Jens (2011), “PPor qué la gimnasia de Ling? El desarrollo de la gimnasia sueca durante el siglo XIX", en Pablo Scharagrodsky (org.), La invención del homo gymnasticus, Buenos Aires, Prometeo, pp. 37-51.

Mayoral, Ángel (2012), "La Escuela Central de Profesores y Profesoras de Gimnástica, en el inicio de una profesión", Revista Athlos, vol. III, año II, pp. 7-36.

Miranda, Marisa (2012), "La Argentina en el escenario eugénico internacional", en Marisa Miranda y Gustavo Vallejo (org.), Una historia de la eugenesia. Argentina y las redes biopolíticas internacionales 1912-1945, Buenos Aires, Biblos, pp. 19-64.

Orbuch, Iván (2016), Peronismo y Educación Física. Políticas públicas entre 1946 y 1955, Buenos Aires, Miño y Dávila.

Pineau, Pablo (dir.) (2014), Escolarizar lo sensible. Estudios sobre estética escolar (1870-1945), Buenos Aires, Teseo.

Ron, Osvaldo (2019), Nociones de cuerpo educado en la formación superior. El caso del Plan de Estudios de Profesorado y Licenciatura en Educación Fisica, UNLP (2000-2017), Buenos Aires, Doctorado en Ciencias Sociales, Universidad Nacional de La Plata.

Scharagrodsky, Pablo (2015), "El Sistema Argentino de 
Educación Física. Entre el cientificismo, la higienización, el eclecticismo y la argentinidad", Revista Brasileira de Ciências do Esporte, vol. 37, pp. 158-164.

Scharagrodsky, Pablo (2014), "El discurso médico y su relación con la invención del oficio de 'educador físico': entre la heteronomía solapada y la autonomía vigilada (Argentina, 1901-1931)", en Pablo Scharagrodsky (comp.), Miradas médicas sobre la cultura fisica en Argentina, 1880-1970, Buenos Aires, Editorial Prometeo, pp. 101148.

Scharagrodsky, Pablo (2011), "La constitución de la Educación Física escolar en la Argentina. Tensiones, conflictos y disputas con la matriz militar en las primeras décadas del siglo XX", en Pablo Scharagrodsky (comp.), La invención del 'homo gymnasticus'. Fragmentos históricos sobre la educación de los cuerpos en movimiento en Occidente, Buenos Aires, Editorial Prometeo, pp. 441-475.

Scharagrodsky, Pablo (2004), "La Educación Física Escolar Argentina (1940-1990) De la fraternidad a la complementariedad", Revista Anthropologica, vol. XXII, núm. 22, pp. 63-92.

Terigi, Flavia (2000), Carpeta de Psicología Educacional, Bernal, Universidad Virtual de Quilmes-Imprenta U.N.Q.
Toconás, Sergio (2006), "La formación docente en Educación Física en la Provincia de Jujuy. Una introducción al caso del año 1956", en Rodolfo Rozengardt (ed.), Apuntes de historia para profesores de Educación Física, Buenos Aires, Miño y Dávila, pp. 337355.

Torres, César (2011), "La Educación Física en los Estados Unidos (1865-1945)", en Pablo Scharagrodsky (org.), La invención del homo gymnasticus, Buenos Aires, Prometeo, pp. 253-277.

Vigarello, Georges y Richard Holt (2005), "El cuerpo cultivado: gimnastas y deportistas en el siglo XIX", Alain Corbin, Jean-Jacques Courtine y Georges Vigarello (dirs.), Historia del cuerpo. De la Revolución francesa a la Gran guerra (Vol. II), Madrid, Taurus, pp. 295-354.

Villa, Alicia (2011), "Currículum, Educación Física y formación del profesorado: El caso del profesorado de Educación Física de la Universidad Nacional de La Plata", Ágora para la educación fisica y el deporte, vol. 13, núm. 3, pp. 321-340.

Villa, Alicia (2007), "La conformación de la profesionalidad de la educación física desde la formación de profesores universitarios", Revista Brasileira de História da Educaçao, vol. 7, núm. 2, Brasil, pp. 117-144.

Cómo citar este artículo:

Levoratti, Alejo y Pablo Scharagrodsky (2021), "Notas para una historia de las instituciones argentinas de formación de docentes en Educación Física durante el siglo xx", Revista Iberoamericana de Educación Superior (RIES), vol. XII, núm. 35, pp. 92-110, DOI: https:// doi.org/10.22201/iisue.20072872e.2021.35.1084 [Consulta: fecha de última consulta]. 